\title{
Aggressive resection at the infiltrative margins of glioblastoma facilitated by intraoperative fluorescein guidance
}

\author{
*Justin A. Neira, MD, ${ }^{1}$ Timothy H. Ung, MD, ${ }^{1}$ Jennifer S. Sims, PhD, ${ }^{1}$ Hani R. Malone, MD, ${ }^{1}$ \\ Daniel S. Chow, MD, ${ }^{2}$ Jorge L. Samanamud, ${ }^{1}$ George J. Zanazzi, MD, PhD, ${ }^{4}$ Xiaotao Guo, PhD, ${ }^{3}$ \\ Stephen G. Bowden, BM, ${ }^{1}$ Binsheng Zhao, DSc, ${ }^{3}$ Sameer A. Sheth, MD, PhD, ${ }^{1}$ \\ Guy M. McKhann II, MD, ${ }^{1}$ Michael B. Sisti, MD, ${ }^{1}$ Peter Canoll, MD, PhD, ${ }^{4}$ Randy S. D'Amico, MD, ${ }^{1}$ \\ and Jeffrey N. Bruce, MD'
}

Departments of ${ }^{1}$ Neurological Surgery, ${ }^{2}$ Neuroradiology, ${ }^{3}$ Radiology, and ${ }^{4}$ Pathology and Cell Biology, Columbia University Medical Center, New York, New York

OBJECTIVE Extent of resection is an important prognostic factor in patients undergoing surgery for glioblastoma (GBM). Recent evidence suggests that intravenously administered fluorescein sodium associates with tumor tissue, facilitating safe maximal resection of GBM. In this study, the authors evaluate the safety and utility of intraoperative fluorescein guidance for the prediction of histopathological alteration both in the contrast-enhancing $(\mathrm{CE})$ regions, where this relationship has been established, and into the non-CE (NCE), diffusely infiltrated margins.

METHODS Thirty-two patients received fluorescein sodium (3 mg/kg) intravenously prior to resection. Fluorescence was intraoperatively visualized using a Zeiss Pentero surgical microscope equipped with a YELLOW 560 filter. Stereotactically localized biopsy specimens were acquired from CE and NCE regions based on preoperative MRI in conjunction with neuronavigation. The fluorescence intensity of these specimens was subjectively classified in real time with subsequent quantitative image analysis, histopathological evaluation of localized biopsy specimens, and radiological volumetric assessment of the extent of resection.

RESULTS Bright fluorescence was observed in all GBMs and localized to the CE regions and portions of the NCE margins of the tumors, thus serving as a visual guide during resection. Gross-total resection (GTR) was achieved in $84 \%$ of the patients with an average resected volume of $95 \%$, and this rate was higher among patients for whom GTR was the surgical goal (GTR achieved in $93.1 \%$ of patients, average resected volume of $99.7 \%$ ). Intraoperative fluorescein staining correlated with histopathological alteration in both CE and NCE regions, with positive predictive values by subjective fluorescence evaluation greater than $96 \%$ in NCE regions.

CONCLUSIONS Intraoperative administration of fluorescein provides an easily visualized marker for glioma pathology in both CE and NCE regions of GBM. These findings support the use of fluorescein as a microsurgical adjunct for guiding GBM resection to facilitate safe maximal removal.

https://thejns.org/doi/abs/10.3171/2016.7.JNS16232

KEY WORDS glioblastoma; fluorescein; fluorescence-guidance; fluorescence-guided resection; high-grade glioma; oncology

\footnotetext{
ABBREVIATIONS 5-ALA = 5-aminolevulinic acid; BBB = blood-brain barrier; CE = contrast enhancing; CUMC = Columbia University Medical Center; EOR = extent of resection; GBM = glioblastoma; GTR = gross-total resection; HGG = high-grade glioma; $n-A N O V A=$ multiway ANOVA; NCE = non-contrast enhancing; OS = overall survival; PFS = progression-free survival; $\mathrm{PPV}=$ positive predictive value; $\mathrm{ROI}=$ region of interest; $\mathrm{STR}=$ subtotal resection; UD = undetermined; WHO = World Health Organization; Y560 = YELLOW 560.
}

SUBMITTED February 6, 2016. ACCEPTED July 12, 2016.

INCLUDE WHEN CITING Published online October 7, 2016; DOI: 10.3171/2016.7.JNS16232.

* Drs. Neira and Ung have contributed equally to this work. Drs. D'Amico and Bruce share senior authorship of this work. 
$\mathrm{S}$ AFE maximal resection is an independent predictor of glioblastoma (GBM) prognosis, ${ }^{7,14,25}$ with a mean overall patient survival of 14.6 months even in the context of adjuvant radiotherapy and temozolomide. ${ }^{35}$ In an effort to achieve safe gross-total resection (GTR), MRIbased neuronavigation - combining spatial precision, differential identification of tumor and nontumor tissues, and neuroanatomical reference-has become the standard of care in modern glioma surgery. Yet, persistent difficulties with intraoperative brain shift, reliance on accurate registration, and reliance on contrast enhancement to identify tumor can hinder real-time identification of resectable tumor, contributing to residual disease at the resection margins. ${ }^{35}$ The infiltrative nature of GBM presents a particular challenge as traditional imaging modalities do not precisely delineate the tumor-brain interface or identify regions of nonenhancing tumor cells. ${ }^{3,6}$ The development of surgical adjuncts that allow for intraoperative identification of nonenhancing tumor may increase cytoreductive efforts with real benefits to prognosis.

The search for real-time intraoperative guidance during microsurgery has fostered the exploration of fluorescent dye labeling to facilitate discrimination of neoplastic tissue from healthy structures and increase safely resected volumes. Administration of 5-aminolevulinic acid (5-ALA), ${ }^{32,33}$ which is incorporated into the fluorescent protoporphyrin IX of tumor cells, facilitates compelling increases in short-term progression-free survival (PFS) and resection volumes, as well as decreases in the rate of subtotal tumor resection. ${ }^{28}$ However, questions regarding the tumor specificity of 5-ALA metabolism ${ }^{15}$ and the dye's phototoxicity risk, ${ }^{12}$ high cost, and lack of Food and Drug Administration approval have limited its use in the United States. Fluorescein sodium, an easily administered, widely marketed, biosafe green-fluorescing dye, has been used as an adjunct for the resection of intracranial tumors since the $1940 \mathrm{~s},,^{20,21}$ as well as in numerous other medical applications. ${ }^{22}$ The extravasation of fluorescein ${ }^{9}$ via disruptions in the blood-brain barrier (BBB) leads to accumulation in the extracellular space and high fluorescence. ${ }^{9,29}$ In comparison, dye leakage is minimal in tissue with an intact $\mathrm{BBB}$ and readily cleared from brain tissue. ${ }^{23}$ With sufficiently high doses of dye, fluoresceinguided resection has been associated with increased rates of complete radiographic resection even without the use of a dedicated fluorescence microscope. ${ }^{16,27}$

The emergence of surgical microscopes outfitted with a fluorescein-specific filter has enabled broader application of fluorescein guidance to glioma microsurgery ${ }^{2,17,26}$ with a statistically strong association between fluorescein staining and tumor tissue, as well as reports that fluorescein guidance safely facilitates complete resection of contrast-enhancing (CE) tumor. ${ }^{1,9}$ However, small study groups have offered limited evidence of the added utility of fluorescein guidance, and rigorous assessment of fluorescein as a marker for tumor pathology in regions beyond MRI contrast enhancement has not been performed. ${ }^{2,9}$

In the present study, we evaluate the utility of intraoperative fluorescein imaging for identification of neoplastic tissue, present analytical methods for robust quantification of fluorescein intensity, and assess the correlation of fluorescein staining with histopathological alteration in radiographically localized biopsies, especially as it pertains to regions of nonenhancing tumor. In addition, we corroborate earlier reports of high patient safety, ease of administration, and subjective utility. Our results show that fluorescein is a safe and reliable marker for intraoperative visualization of glioma pathology, both in $\mathrm{CE}$ and non-CE (NCE) regions of GBM.

\section{Methods}

\section{Patient Selection and Operative Protocol}

Thirty-two patients with cerebral lesions suspicious for malignant gliomas were prospectively enrolled to undergo fluorescein-guided resection between October 2013 and October 2014 at the Columbia University Medical Center (CUMC). Inclusion criteria were as follows: 1) age $\geq 18$ years; 2) newly diagnosed, untreated, WHO Grade III or IV glioma suspected given brain MRI studies with and without gadolinium contrast; and 3) known, previously treated, recurrent high-grade glioma (HGG). Exclusion criteria included 1) age $<18$ years, 2) histological diagnosis other than $\mathrm{HGG}, 3$ ) brainstem tumor, 4) multicentric tumor, 5) medical reasons precluding MRI with contrast, 6) renal insufficiency, and 7) hepatic insufficiency. This study includes only patients with WHO Grade IV GBM. Written informed consent was obtained from all patients, and all aspects of this study were performed in accordance with the institutional review board guidelines at CUMC.

Fluorescein sodium 10\% (Alcon Laboratories) was intravenously administered at a dose of $3 \mathrm{mg} / \mathrm{kg}$ following induction of anesthesia and prior to surgical incision. This dosage and timing protocol was selected based on a previous study. ${ }^{24} \mathrm{~A}$ dedicated surgical microscope outfitted with both a fluorescence filter (excitation 460-500 $\mathrm{nm}$, emission 540-690 nm) and unfiltered white-light illumination (OPMI Pentero 900 surgical microscope with YELLOW 560 [Y560] fluorescence visualization kit, Carl Zeiss) allowed switching of the illumination source between the fluorescence excitation source $(2 \times 300 \mathrm{~W}$ xenon) and white light (Superlux LED) throughout the surgery (Fig. 1A and B). This configuration permitted significant portions of each surgery to be performed under the fluorescence filter while observing the fluorescein intensity in real time. Curve Cranial Navigation Software (BrainLab) was used for MRI-based neuronavigation and localization of biopsied tissue.

Patients were evaluated immediately after surgery and on the first postoperative day for adverse events related to fluorescein sodium. Adverse events were graded based on Common Terminology Criteria for Adverse Events version 4.0, and a plausible relationship to fluorescence administration was evaluated. Karnofsky Performance Status was evaluated as part of the routine preoperative examination; postoperative performance was evaluated by a board-certified neurosurgeon, neurologist, or neurooncologist, and the Karnofsky Performance Status score was recorded by a member of the research team on the postsurgery day noted in Table 1 . All patients received standardized postcraniotomy care. Magnetic resonance imaging was performed within 48 hours of surgery. 

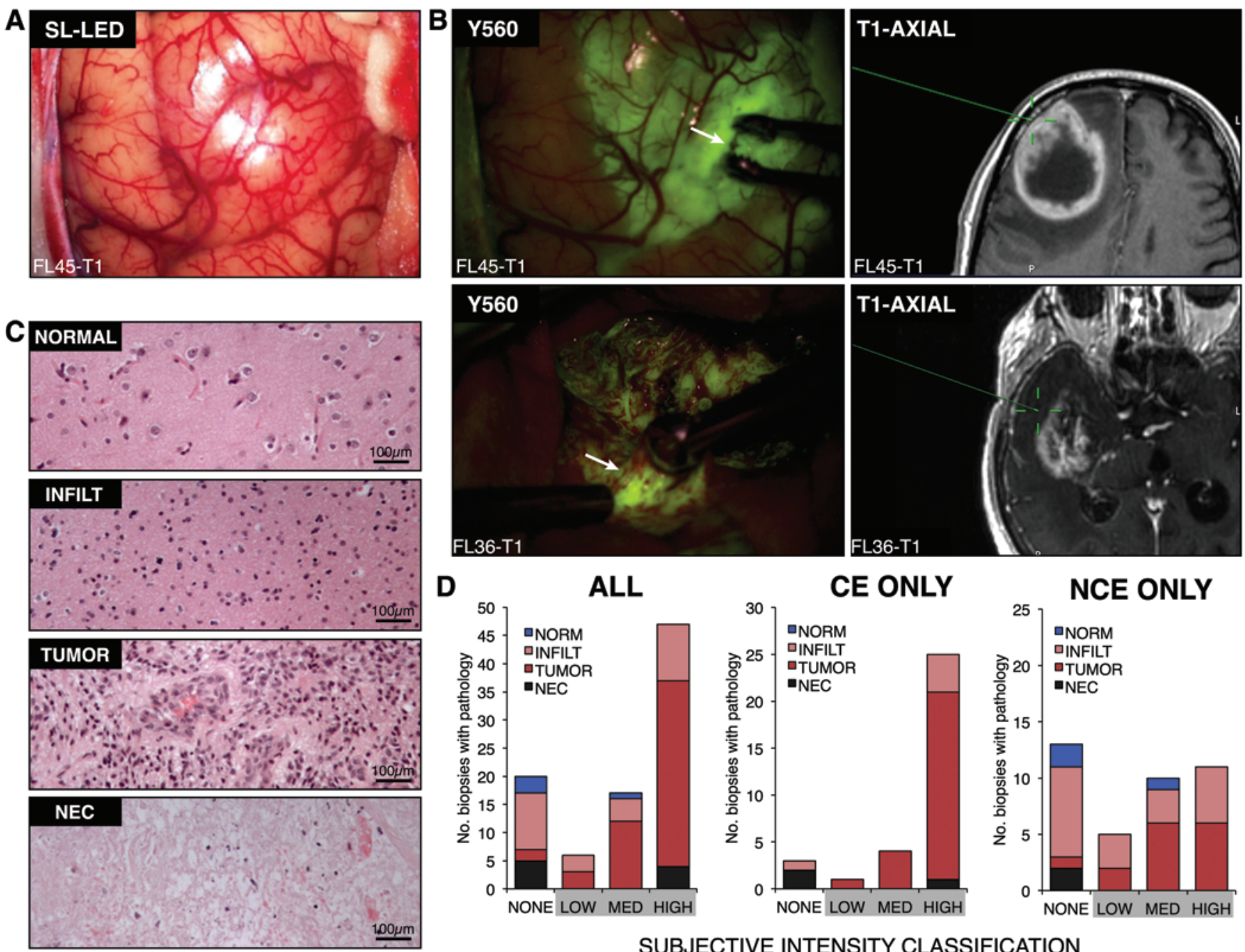

\section{SUBJECTIVE INTENSITY CLASSIFICATION}

FIG. 1. Intraoperatively observable fluorescence intensity associates with radiographic tumor localization and histopathology in CE and NCE regions. Video images were recorded with the Pentero internal camera throughout surgery. A: Representative image of absent fluorescein staining in the region under illumination by the white light diode (SL-LED), captured just prior to Y560 excitation. B: Distinctive yellow-green staining was visible under illumination with the Y560 filter set (left, excitation 460-500 nm, emission $540-690 \mathrm{~nm}$ ) in the same surgical field depicted in panel A and an additional field from an independent surgery (biopsy identification numbers: FL45-T1, FL36-T1), representing the site of biopsies (white arrows) from CE (upper) and NCE (lower) regions, as determined by stereotactic localization of the BrainLab-registered wand held at the biopsy site (green crosshairs) on preoperative MRI (T1, gadolinium contrast, right). C: Biopsy specimens were histopathologically classified as follows: normal appearing brain tissue (NORMAL), glioma-infiltrated tissue (INFILT), frank tumor (TUMOR), and necrotic tissue (NEC). H \& E. D: Subjective assessment of fluorescein intensity was made at the time of acquisition for 90 biopsies from noneloquent locations among 26 of the 32 cases and was confirmed post hoc by video image. The distribution of pathology classifications by fluorescence intensity-subjectively described as none, low, medium, and high-is graphed for all 90 samples (left), for 33 samples radiographically localized to CE regions (center), and for 39 NCE regions (right). Med = medium.

\section{Radiographic Imaging and Assessment of Extent of Resection}

All imaging was performed on a 1.5- or 3-T MRI system with an 8-channel head-array coil (Signa HDxt, GE Healthcare). Axial T1-weighted pre- and postcontrast images and axial FLAIR images were obtained. Volumetric acquisitions were also obtained for all postcontrast images by using a T1-weighted 3D inversion recovery fast spoiled gradient-recalled sequence. Postcontrast images were acquired with a weight-based dose of intravenous gadobenate dimeglumine at $0.2 \mathrm{ml} / \mathrm{kg}$ (MultiHance, Bracco Diag- nostics Inc.). The time between intravenous injection and postcontrast imaging was 5 minutes. All postoperative imaging was performed within 48 hours of resection.

A radiologist blinded to case-control status evaluated extent of resection (EOR) by using computer-assisted volumetric measurements of all pre- and postoperative imaging. Briefly, this semi-automated algorithm combines the region-based active contours and a level set approach and has been shown to provide for reproductive assessment of enhancing tumor burden in postoperative GBM patients. ${ }^{8}$ All measurements were performed with correlation 
TABLE 1. Demographics and biopsies for patients enrolled for fluorescein-guided tumor resection

\begin{tabular}{|c|c|c|c|c|c|c|c|}
\hline Case Type & Tumor Location & Sex & Age (yrs) & Preop KPS & Postop KPS* & No. of Biopsies & Biopsies w/ Time Stamp \\
\hline REC & Lt P & $F$ & 58.1 & 80 & $50(143)$ & 0 & NA \\
\hline GBM & $\mathrm{Lt} T$ & M & 42.1 & 90 & $90(31)$ & 0 & NA \\
\hline GBM & Rt T/P & $\mathrm{F}$ & 46.9 & 90 & $90(11)$ & 3 & $\mathrm{~N}$ \\
\hline GBM & Lt F/T & $\mathrm{F}$ & 72.4 & 80 & $70(31)$ & 3 & $\mathrm{Y}$ \\
\hline REC & Rt P & M & 60.4 & 100 & $90(10)$ & 3 & $\mathrm{~N}$ \\
\hline GBM & Lt T & M & 62.3 & 90 & $90(7)$ & 3 & Y \\
\hline GBM & $\mathrm{Lt} F$ & M & 52.7 & 50 & $90(9)$ & 3 & $\mathrm{Y}$ \\
\hline GBM & Rt T & $\mathrm{F}$ & 66.2 & 90 & $85(22)$ & 5 & $\mathrm{Y}$ \\
\hline GBM & Lt T & $\mathrm{F}$ & 70.2 & 80 & $60(113)$ & 2 & $\mathrm{~N}$ \\
\hline GBM & Rt F & $\mathrm{F}$ & 82.9 & 90 & 90 (112) & 3 & $\mathrm{~N}$ \\
\hline REC & Lt O/T/P & $\mathrm{F}$ & 58.9 & 80 & 70 (10) & 0 & Y \\
\hline GBM & $\mathrm{Lt} \mathrm{T}$ & M & 80.4 & 90 & $90(7)$ & 3 & $Y$ \\
\hline REC & $\mathrm{Lt} F$ & $\mathrm{~F}$ & 39.1 & 90 & $90(1)$ & 2 & $\mathrm{~N}$ \\
\hline GBM & Lt FP & $M$ & 63.9 & 90 & $90(38)$ & 5 & NA \\
\hline REC & Rt P & $\mathrm{F}$ & 60.2 & 80 & $80(9)$ & 1 & Y \\
\hline GBM & Rt T & M & 88.1 & 80 & $80(13)$ & 6 & $\mathrm{~N}$ \\
\hline GBM & Rt O & M & 52.2 & 90 & $100(8)$ & 3 & $Y$ \\
\hline REC & Rt F & $\mathrm{F}$ & 42.2 & 80 & 80 (1) & 5 & $\mathrm{~N}$ \\
\hline GBM & Rt T & $\mathrm{F}$ & 90.7 & 90 & $90(8)$ & 8 & Y \\
\hline GBM & Rt F & $M$ & 70.1 & 85 & $65(2)$ & 3 & Y \\
\hline REC & Rt T & M & 43.2 & 90 & 80 (1) & 3 & Y \\
\hline GBM & $\mathrm{Lt} F / T$ & M & 67.5 & 80 & 80 (1) & 0 & $Y$ \\
\hline REC & Rt P & $\mathrm{F}$ & 78.2 & 60 & $60(1)$ & 0 & $Y$ \\
\hline REC & Rt $P$ & $\mathrm{~F}$ & 76.5 & 45 & $45(1)$ & 0 & $Y$ \\
\hline GBM & Lt T & $F$ & 87.3 & 85 & $85(1)$ & 4 & $Y$ \\
\hline REC & Rt T & $F$ & 54.1 & 90 & $85(1)$ & 3 & NA \\
\hline GBM & Rt F & $\mathrm{F}$ & 53.9 & 90 & 75 (1) & 2 & NA \\
\hline REC & Rt F & $M$ & 72.2 & 90 & $90(1)$ & 3 & NA \\
\hline GBM & $\mathrm{Lt} F$ & $M$ & 24.2 & 90 & 70 (1) & 4 & $Y$ \\
\hline REC & Rt T/P & $M$ & 77.0 & 70 & $60(2)$ & 2 & $Y$ \\
\hline GBM & Rt P & M & 66.7 & 90 & $70(1)$ & 3 & $\mathrm{Y}$ \\
\hline GBM & Rt F & M & 84.1 & 90 & $70(4)$ & 5 & Y \\
\hline All cases $(n=32)$ & & $16 \mathrm{M}, 16 \mathrm{~F}$ & 63.9 & $83.3 \pm 12.0$ & $78.4 \pm 13.4$ & $90(26) \dagger$ & $73(20) \dagger$ \\
\hline GBM cases $(n=20)$ & & $12 \mathrm{M}, 8 \mathrm{~F}$ & 66.2 & $85.5 \pm 9.3$ & $81.5 \pm 10.9$ & $60(18) \dagger$ & $51(16) \dagger$ \\
\hline REC cases $(n=12)$ & & $4 \mathrm{M}, 8 \mathrm{~F}$ & 60.0 & $80.0 \pm 15.0$ & $73.0 \pm 16.0$ & $30(8) \dagger$ & $22(4) \dagger$ \\
\hline
\end{tabular}

$\mathrm{F}=$ frontal; GBM = primary GBM; KPS = Karnofsky Performance Status; $N=$ no; NA = timestamp data not available from recorded video; $\mathrm{O}=0 \mathrm{cccipital} ; \mathrm{P}=$ parietal;

REC = recurrent GBM; $T=$ temporal; $Y=$ yes.

* Parentheses denote number of days after surgery when KPS score was recorded.

$\dagger$ Value in parentheses indicates the number of patients in whom the biopsies were obtained.

to precontrast $\mathrm{T} 1$ images to avoid $\mathrm{T} 1$ shortening effects from postsurgical changes (for example, blood products). Residual tumor was defined as a single area of enhancement measuring $0.175 \mathrm{~cm}^{3}$ or more. Extent of resection was gross total (GTR) if $<0.175 \mathrm{~cm}^{3}$ or subtotal (STR) if $>$ $0.175 \mathrm{~cm}^{3}$ of residual, singular finite volume of contrast enhancement was present postoperatively (Fig. 2). ${ }^{32}$ Prior to EOR analysis, patients were classified as "GTR amenable" based on the operative and preoperative notes, and all radiologists participating in the MRI-based EOR assessment were blinded to the surgical goal. It was therefore possible to achieve extensive resections even in cases in which functional eloquence, structural accessibility, or other factors led the neurosurgeon to refrain from stating GTR as an explicit surgical goal. Likewise, it was possible for a very high percentage of $\mathrm{CE}$ volume to be resected without the absolute residual volume falling below $0.175 \mathrm{~cm}^{3}$.

A retrospective control cohort consisted of 32 (WHO Grade IV) GBM cases resected at CUMC under conditions equivalent to those of the fluorescein-guided cohort except for fluorescein use (facilities, staff, availability of radiographic neuronavigation). A member of the research team and a neuroradiologist blinded to the study selected the control cases to closely approximate the following fea- 

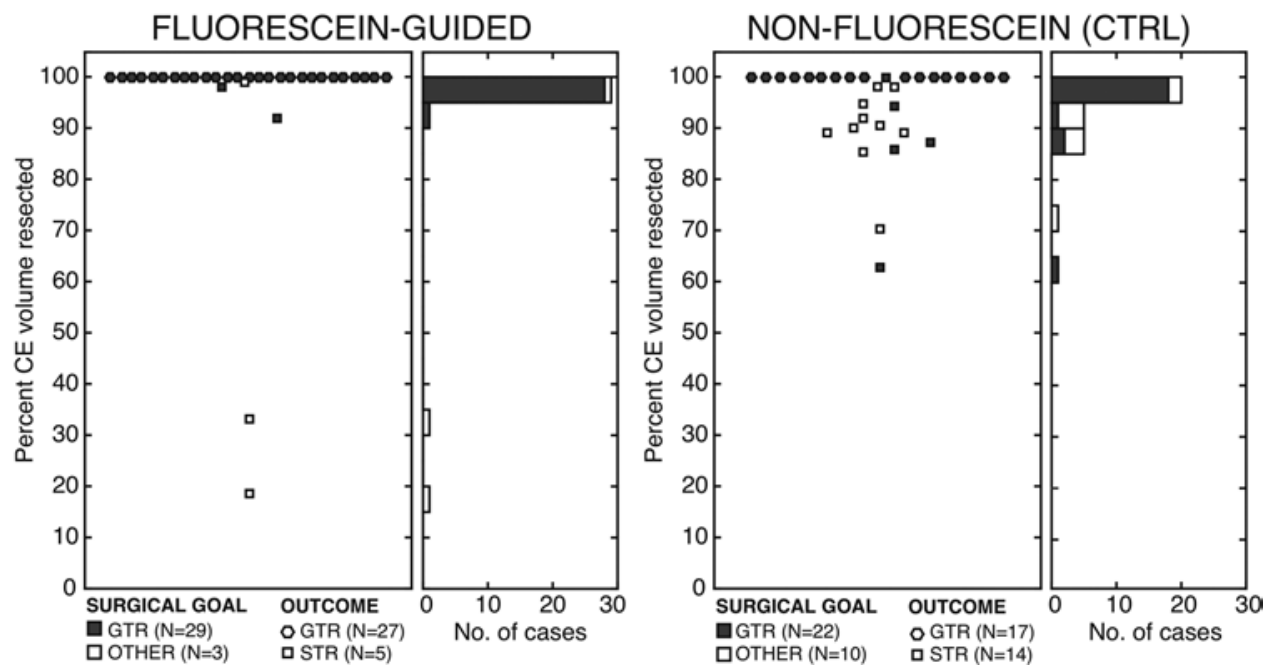

FIG. 2. Fluorescein guidance facilitates extensive resection of CE volume in GBM. Resected CE volume was calculated for fluorescein-guided surgeries (32 cases), as well as matched control cohort patients (32 cases), by subtracting residual CE volume on postoperative T1-weighted MRI from the preoperative volume. Left: Distribution of CE volume resected among fluorescein-guided cases, in 29 of which GTR was the stated surgical goal (shaded in black, average resected volume 99.7\%). Right: Distribution of CE volume resected among nonfluorescein cases, in 22 of which GTR was the stated surgical goal (shaded in black, average resected volume $96.8 \%$ ). Outcome of GTR (hexagons) reflects the presence of $<0.175 \mathrm{~cm}^{3}$ residual CE volume. CTRL $=$ control.

tures of the fluorescein-guided cohort (Table 1): age (60.5 \pm 15.9 years), sex (17 male, 15 female), presentation (20 primary GBMs, 12 recurrent GBMs), and tumor location. The surgical goal as well as GTR amenability was retrospectively obtained from the operative notes, as in the fluorescein-guided cases.

\section{Biopsy, Subjective Fluorescence, and Histopathology of Stereotactically Localized Sites}

Stereotactically localized biopsy specimens were obtained, and their fluorescence intensity was subjectively labeled as "none," "low," "medium," and "high" based on the surgical team's visual inspection of the real-time digital video from the intraoperative microscope (Fig. 1A and $\mathrm{B}$ and Table 2). Tissue biopsies were obtained from both CE (33 cases) and NCE (39 cases) regions as indicated by neuronavigation, and this status or the fact that the biopsy was "undetermined" (UD) and/or necrotic (NEC; hypointense core circumscribed by CE) by MRI (UD/NEC, 18 cases) was recorded. "Undetermined" was used in cases in which radiographic localization was unavailable or the accuracy of registration was compromised. For downstream quantification of fluorescence, a time-stamped digital video still was captured using the Pentero internal camera, permitting clear visualization of biopsied tissue in 26 of the 32 enrolled cases.

Biopsy tissue was fixed in 10\% formalin for histological and immunohistochemical analysis (Fig. 1C). Two neuropathologists, each blinded to case and sample identity, analyzed H \& E-stained, formalin-fixed paraffinembedded tissue. Tissue was classified as frank tumor, glioma-infiltrated brain, necrotic, or no diagnostic abnormality. In cases in which there was disagreement on initial assessment of the biopsy, the pathologists conferred and agreed on the histological category to be assigned. For statistical analysis of sensitivity, specificity, and positive pre- dictive value (PPV), the 3 categories showing histopathological alterations (tumor, glioma infiltration, or necrosis) were compared with specimens showing no histological abnormalities. The $\mathrm{H} \& \mathrm{E}$-stained slides were scanned to determine the area of highest cellular density. Representative photomicrographs were acquired using ImageJ 1.46r software at a magnification $\times 100$ (Fig. 1C; Olympus BX43 light microscope, Olympus DP-26 camera).

\section{Quantification of Fluorescein Signal and Statistical Relationships}

Fluorescence intensity quantification was performed on digital still-frame images taken from the time of tissue biopsy (see above) and processed to isolate green fluorescent emission using Photoshop 2014 (Adobe). Briefly, a broad rectangular area encompassing the illuminated surgical field was selected (Fig. 3) and used to form a histogram of pixel intensities of the entire surgical field (range 0-255). Additionally, each individual biopsy specimen was delineated, and a circular region of interest (ROI; 0.5 times

TABLE 2. Comparison of fluorescence intensity classification by intraoperative (subjective) description and by retrospective quantitative (objective) image analysis: number of biopsies

\begin{tabular}{crrrrr}
\hline Subjective & \multicolumn{5}{c}{ Objective Fluorescence Intensity } \\
\cline { 2 - 6 } $\begin{array}{c}\text { Fluorescence } \\
\text { Intensity }\end{array}$ & None & Low & Med & High & Total \\
\hline None & 10 & 5 & 4 & 1 & 20 \\
\hline Low & 2 & 1 & 3 & 0 & 6 \\
\hline Med & 3 & 7 & 4 & 3 & 17 \\
\hline High & 0 & 6 & 24 & 17 & 47 \\
\hline Total & 15 & 19 & 35 & 21 & 90 \\
\hline
\end{tabular}

Med $=$ medium 

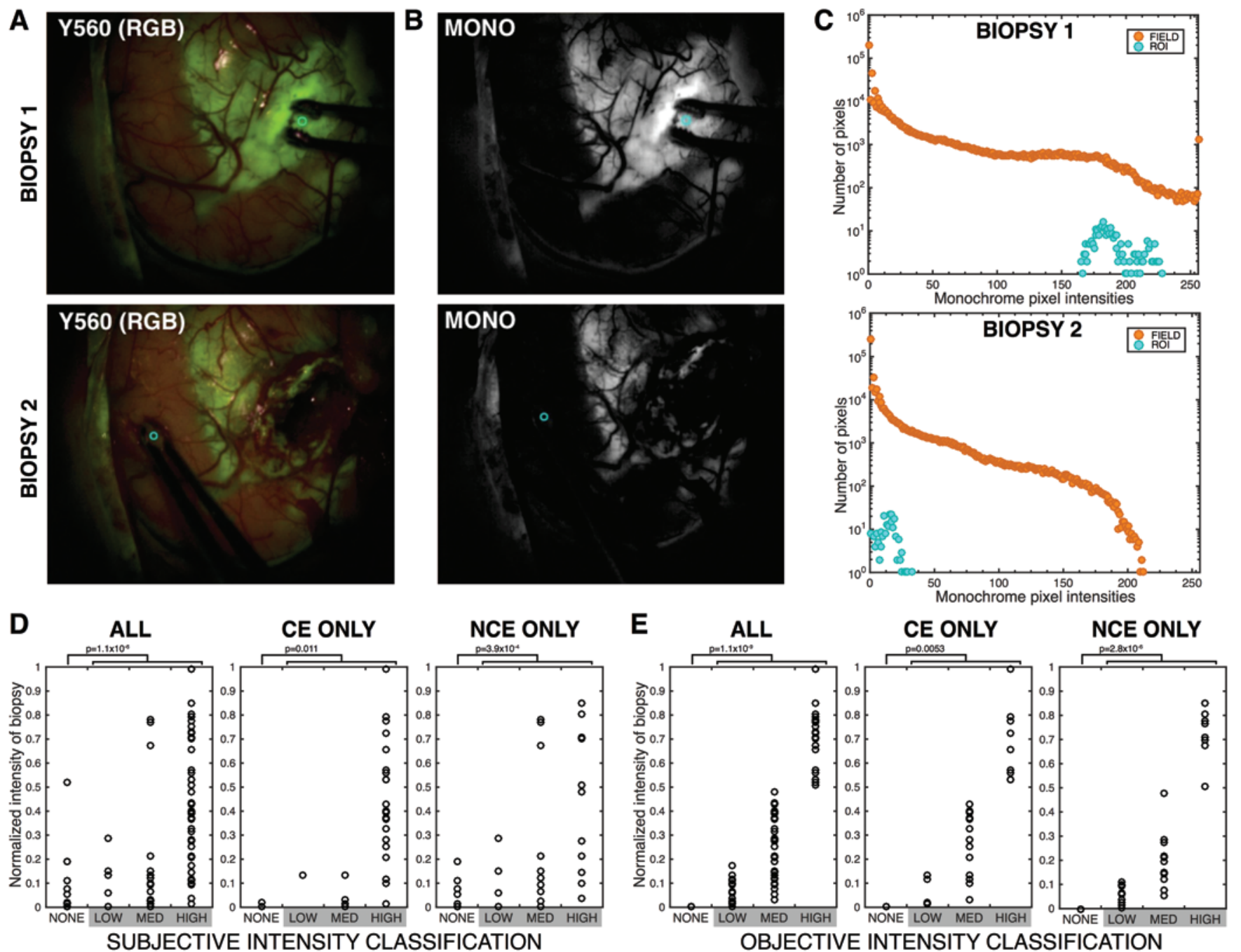

FIG. 3. Quantification of fluorescence intensity by postoperative image analysis and association of objective classification with histopathological diagnosis. A: RGB images captured with the Pentero microscope internal camera under illumination through the Y560 filter set (2 representative biopsies from a single patient are depicted). Regions of interest were specified to represent the entire illuminated field (whole rectangular panel), and the biopsy (circular ROI approximately half the diameter of the distance between the forceps [cyan]). B: The monochrome green channel (MONO) was extracted from these images, and the monochrome pixel intensities for each ROI were exported. C: Histograms for the ROls in Biopsy 1 and Biopsy 2 (cyan) and their accompanying surgical fields (orange). D: The mean monochrome pixel intensity of the ROI for all histopathologically classified biopsies was normalized to their fields (see Methods). The relationship between the normalized fluorescence intensity of these biopsies and the subjective classification of its fluorescence made at the time of surgery is plotted for all biopsies ( 90 biopsies), CE (33 biopsies), and NE (39 biopsies). Significant differences were observed in the normalized intensity values of biopsies subjectively classified as FL- (none) and FL+ (low, med, high). E: Normalized intensity of biopsies was objectively binned, with categories analogous to those in the subjective bins, using thresholds for background, low, medium, and high fluorescence (see Methods) based on the pixel distribution of each field. The relationship between normalized fluorescence intensity and the objective classification is plotted for all samples (90 biopsies), for those radiographically localized to CE regions (33 biopsies), and for those from NCE regions (39 biopsies).

the diameter of the biopsy specimen) including the area of highest intensity was processed to yield a histogram of pixel intensities. Results were exported and analyzed (described below) using home-coded programs written in Python 2.7.5, whose source code and detailed description are available at https://github.com/JNBlab/FL_image_analysis. During the development of this analysis platform, circular ROIs 1 and 2 times the biopsy specimen diameter, as well as closely traced ROIs masking the shape of the biopsied specimen, were explored, and 0.5 times the diam- eter was found to produce robust values across images and test users and was therefore integrated into our workflow. Examples of these alternative ROI strategies are available in the same repository.

Briefly, quantitation of the fluorescence intensity of each biopsy ROI by mean pixel intensity enabled its characterization by 2 types of metrics: an "objective intensity" bin analogous to the subjective bin and a "normalized intensity." For each microscopic field, a large rectangular ROI approximately comprising the entire illuminated sur- 
gical field was selected. The median pixel intensity (up to $10 \%$ of the total range of pixel intensities) was considered background. The mean intensity of pixels in the highest $5 \%$ of intensities was considered the maximum ("max") intensity to allow for variation in overall illumination. The raw intensity of each biopsy was normalized to the range between the background threshold and the max intensity for the field in which it was imaged, with raw intensities below this range set equal to 0 and those above this range set equal to 1 . The objective fluorescence intensity bins (none, low, medium, and high-analogous to those used for the subjective classification) were determined using the median pixel intensity (background) as the lower bound for the "low" range, the mean intensity of pixels above background as the lower bound for the "mid" range, and the midpoint between background and max intensity $\left(\left[i_{1}\right.\right.$ $\left.+\mathrm{i}_{2}\right] / 2$ ) as the lower bound for the "high" range (Table 2). The median, mean, and midpoint fluorescence intensities of the illuminated fields were not correlated with the time elapsed following fluorescein administration (Spearman correlation between time elapsed and median pixel intensity over all biopsies with time stamps 73 cases, rho $=0.03$, $\mathrm{p}=0.78$ ). Additional correlations, Wilcoxon rank-sum, and multivariate ANOVA ("anovan," using fluorescence intensity and time as continuous variables and $\mathrm{CE}$ vs $\mathrm{NCE}$ and pathological diagnosis as classifications) tests were calculated using MATLAB 2014b (MathWorks).

To show that stratification of biopsy samples according to normalized fluorescence intensity reflected the same groupings as the none, low, medium, and high classifications, especially those observed subjectively at the time of acquisition and which best reflect how fluorescence intensity has been assessed in prior studies, we treated normalized fluorescence intensity as a continuous variable and calculated the significance of the difference in the distributions of these values among biopsies classified as having none, low, medium, or high fluorescence, either subjectively or objectively. Analysis was performed in MATLAB 2014b ("ttest2," unpaired), and similar results were obtained using the Wilcoxon rank-sum function ("ranksum"). Tabulated data for each biopsy, including its subjective, objective, and normalized fluorescence intensity, pathology classification, and radiographic localization, along with our analysis programs, are available at https:// github.com/JNBlab/FL_image_analysis.

\section{Results}

\section{Study Enrollment and Safety}

The 32 patients prospectively enrolled with histologically confirmed GBM (20 primary, 12 recurrent) ranged in age from 39-90 years (mean 63.9 years) and included 16 females and 16 males (Table 1). Preoperatively, all subjects were deemed stable and suitable for intravenous administration of fluorescein according to clinical laboratory criteria, and all tumor sites were deemed potentially amenable to safe tumor resection (GTR or STR). A single dose of fluorescein sodium $(3 \mathrm{mg} / \mathrm{kg}$ ) delivered intravenously after the induction of anesthesia was found to be safe in GBM patients undergoing tumor resection. No allergic reactions and/or adverse events associated with the use of fluores- cein sodium were observed, while pre- and postoperative neurological performance was consistent with that exhibited following GTR of malignant glioma. ${ }^{19,20}$

\section{Impact of Differential Fluorescein Intensity on Aggressive Resection and Operative Experience}

Intraoperative detection of a fluorescein signal was achieved using the Zeiss Pentero system in combination with BrainLab neuronavigation: application of the Y560 long pass filter set revealed characteristic yellow-green fluorescence (subjective assessment) of the dura mater in all cases, whereas white light illumination of these tissues did not (Fig. 1A). High intensity fluorescence coincided well with areas of $\mathrm{CE}$ tumor as depicted by neuronavigation (Fig. 1B upper). Notably, fluorescence was also observed outside the $\mathrm{CE}$ regions in many tumors (Fig. 1B lower). Necrotic tissue exhibited variable levels of fluorescence, from bright to little or no fluorescence. In all cases, illumination of the operative field via the Y560 filter allowed simultaneous differential visualization and awareness of microsurgical anatomy and tissue fluorescence, permitting anatomically guided precision and clear identification of fluorescent tissue (Fig. 1B upper left).

Given the potential for fluorescein guidance to facilitate safe, aggressive resection of GBM, we evaluated its impact on EOR. Subtractive volumetry of pre- and postoperative MRI for the 32 fluorescein-guided cases was compared with that for retrospective case-matched controls (32 cases). Two end points were evaluated: the percentage of patients in whom GTR was achieved and the mean percentage of CE tumor volume resected. Grosstotal resection was defined as the presence of $<0.175 \mathrm{~cm}^{3}$ of residual, singular, finite $\mathrm{CE}$ volume. ${ }^{32}$ For cases that the surgeon had deemed GTR amenable, GTR was achieved in $93.1 \%$ (27 of 29; Fig. 2 left) of the fluorescein-guided cases compared with $77.3 \%$ (17 of 22 ; Fig. 2 right) of the control cases. Among these GTR-amenable patients, the mean percent of resected CE tumor volume was $99.7 \% \pm$ $1.5 \%$ in fluorescein-guided cases versus $96.8 \% \pm 8.6 \%$ in control cases. This trend, which approaches significance ( $\mathrm{p}=0.098$, Wilcoxon rank-sum test), suggests that fluorescein facilitates more complete tumor resection. Accordingly, all 4 lead surgeons found fluorescein to be useful as an intraoperative tool for assisting resection in all cases.

\section{Correlation of Subjective Fluorescence Intensity With Histopathology in CE and NCE Regions of GBM}

From 26 of the 32 independent surgeries, 90 stereotactic biopsies at the time of resection were subjectively binned by the surgical team into 4 categories of fluorescein intensity (none 20 cases, low 6 cases, medium 17 cases, or high 47 cases; Table 1), and categories were confirmed by postoperative review of the video images (Fig. 1B). Any discernable subjective fluorescence (low/med/high) assessed subjectively by visual inspection was considered "positive" (FL+), and "none" was considered "negative" (FL-). Formalin-fixed paraffin-embedded sections of these biopsy specimens were stained with $\mathrm{H} \& \mathrm{E}$, and 2 neuropathologists, each blinded to both fluorescence and radiographic correlates, reviewed and assigned the specimens into 1 of 4 histopathological categories: frank tumor, 
TABLE 3. Positive predictive values of subjective intensity classifications for objective classification

\begin{tabular}{ccccc}
\hline \multirow{2}{*}{$\begin{array}{c}\text { Objective Intensity } \\
\text { (observed) }\end{array}$} & \multicolumn{4}{c}{ Subjective Fluorescence Intensity } \\
\cline { 2 - 5 } & None & Low & Med & High \\
\hline None & 0.667 & 0.133 & 0.200 & 0.000 \\
\hline Low & 0.263 & 0.053 & 0.368 & 0.316 \\
\hline Med & 0.114 & 0.086 & 0.114 & 0.686 \\
\hline High & 0.048 & 0.000 & 0.143 & 0.810 \\
\hline
\end{tabular}

glioma-infiltrated brain, necrotic, or no diagnostic abnormality (normal tissue; Fig. 1C). Tumor-containing biopsies included frank tumor, glioma infiltration, and necrosis, as determined histopathologically. With these parameters, subjective fluorescence intensity provided a sensitivity of 0.756 and a specificity of 0.750 for tumor features across all analyzed biopsy samples regardless of radiographic localization (Fig. 1D) and a PPV for tumor pathology of $98.6 \%$.

Just as the utility of intraoperative fluorescein guidance must be considered in the context of neuroanatomical awareness, we evaluated its synergy with radiographic localization. Among biopsies from radiographic locations that were definitively CE (33 cases), sensitivity of fluorescence for tumor pathology was 0.879 . Specificity could not be calculated given the lack of true-negative or false-positive samples; as expected, no CE biopsies were classified as normal by histopathology (Fig. 1D). Among samples from NCE regions (39 cases), subjectively classified positive fluorescein intensity provided a sensitivity of 0.694 , specificity of 0.667 , and a PPV of $96.2 \%$ for biopsies showing histopathological alterations.

\section{Quantitative Analysis of Fluorescein Intensity}

To establish an objective measure of fluorescein levels, we calculated the mean monochrome intensity of each biopsy specimen using an image captured with the Pentero internal video camera upon the specimen's resection (Fig. $3 \mathrm{~A}$ and $\mathrm{B}$ ) and compared it to the distribution of pixel intensities in the surgical field (Fig. 3C). Objective intensity was classified as none, low, medium, and high, which corresponded well with assignments made subjectively during surgery (Tables 2-4).

Although fluorescein intensity at any detectable level subjectively or objectively was strongly predictive of tumor infiltration, we sought to refine our measurement of the biopsy fluorescence intensities beyond the high, medium, and low bins and identify a quantitative correlate with
TABLE 4. Positive predictive values of objective intensity classifications for subjective classification

\begin{tabular}{ccccc}
\hline \multirow{2}{*}{$\begin{array}{c}\text { Subjective Intensity } \\
\text { (observed) }\end{array}$} & \multicolumn{4}{c}{ Objective Fluorescence Intensity } \\
\cline { 2 - 5 } & None & Low & Med & High \\
\hline None & 0.500 & 0.250 & 0.200 & 0.050 \\
\hline Low & 0.333 & 0.167 & 0.500 & 0.000 \\
\hline Med & 0.176 & 0.412 & 0.235 & 0.176 \\
\hline High & 0.000 & 0.128 & 0.511 & 0.362 \\
\hline
\end{tabular}

strong specificity that could be applied to guidance in nonenhancing regions. We normalized the mean pixel intensity of each biopsy to the range of its surgical field-from the background threshold to the maximum intensity-thus correcting for any systemic confounders to the fluorescent illumination of the field. These normalized continuous values stratified according to their subjective (Fig. 3D) and objective (Fig. 3E) classifications. The normalized fluorescence intensity of the biopsies classified as FL- and FL+, either subjectively or objectively, was significantly different between those groups (Table 5), corroborating the utility of this continuous variable to represent the distinctions observed by these classifications. The normalized values also correlated well with their raw values across all pathology classifications (Fig. 4A, overall $\mathrm{R}=0.97$, $\mathrm{p}$ $=3 \times 10^{-54}$ ) and stratified with tumor pathology (Fig. 4B). Among stereotactically localized biopsies, we observed that a threshold of 0.1 normalized fluorescence intensity provided full specificity for glioma-associated pathology in both $\mathrm{CE}$ and NCE regions (Fig. 4C). Although sensitivity was not independently strong $(0.593$ overall, 0.528 for NCE, 0.727 for CE), the high specificity ( 0.750 overall, 1.0 for NCE, not calculated for CE, no normal samples) and high PPV (0.982 overall, 1.0 for CE and NE) of the normalized fluorescence intensity threshold (0.1) support its use as a quantitative correlate of FL+ staining, which predicts the presence of tumor.

The fluorescein emission intensity of each biopsy is influenced by a number of physiological and operative factors, several of which are time dependent or potentially differential based on tissue pathology. To evaluate the impact of such variables on our normalized fluorescence intensity measurement, we performed a multiway ANOVA (n-ANOVA) for time post-fluorescein administration, MRI localization, and pathological diagnosis on the full set of biopsies for which an accurate time stamp was available (73 cases; Table 1). No significant independent relationship was demonstrated between fluorescence and time, MRI lo-

TABLE 5. Difference in normalized fluorescence intensity between objective and subjective classifications of biopsies

\begin{tabular}{|c|c|c|c|c|c|c|}
\hline \multirow[b]{2}{*}{ Categories } & \multicolumn{3}{|c|}{ Subjective } & \multicolumn{3}{|c|}{ Objective } \\
\hline & Overall & CE Only & NCE Only & Overall & CE Only & NCE Only \\
\hline None vs low & $(0.262)$ & $(0.50)$ & (0.349) & $2.6 \times 10^{-7}$ & 0.036 & $2.2 \times 10^{-5}$ \\
\hline Low vs med & $(0.916)$ & $(0.40)$ & $(0.307)$ & $4.3 \times 10^{-7}$ & 0.004 & $1.2 \times 10^{-3}$ \\
\hline Med vs high & $5.6 \times 10^{-4}$ & 0.006 & (0.193) & $5.3 \times 10^{-10}$ & $5.2 \times 10^{-5}$ & $2.5 \times 10^{-4}$ \\
\hline FL- vs FL+ & $1.6 \times 10^{-5}$ & 0.011 & $3.9 \times 10^{-4}$ & $1.1 \times 10^{-9}$ & 0.005 & $2.8 \times 10^{-6}$ \\
\hline
\end{tabular}

$p$ values of 2-sample t-test, uneven variance. Groups without significant difference in distribution indicated by parentheses. 

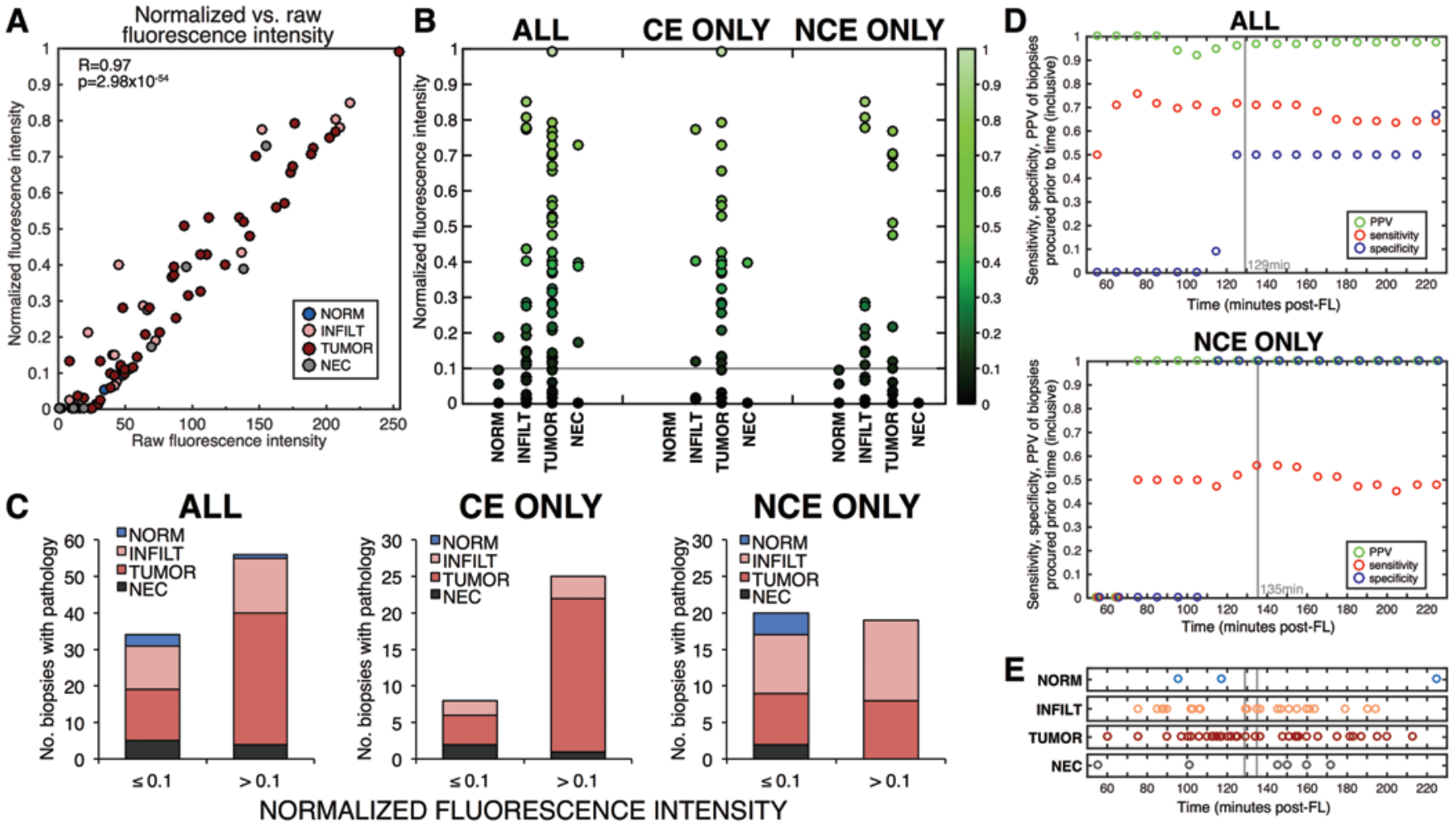

FIG. 4. Normalized fluorescence intensity provides quantitative classification for prediction of histopathological diagnosis. Normalized fluorescence intensity was calculated by normalizing the mean pixel intensity of each biopsy to the range of pixel intensities in its field (see Methods). A: The mean raw fluorescence intensity of the ROI of each biopsy specimen (x-axis) and the normalized fluorescence intensity were strongly correlated (Pearson $R=0.97, p=2.98 \times 10^{-54}$ ). B: The normalized fluorescence intensity of each biopsy specimen is plotted according to its histopathological classification: normal (NORM), tumor infiltrated (INFILT), frank tumor (TUMOR), or necrotic (NEC). All biopsies are plotted together (90 cases), for CE only (33 cases), and for NCE only (39 cases), in a color representing its normalized fluorescence intensity (color bar). Gray line indicates normalized intensity of 0.1. C: The distribution of histopathological classification (NORM, INFILT, TUMOR, or NEC) between fluorescence intensity $\leq 0.1$ and > 0.1 was graphed for all biopsies (90 cases), CE biopsies (33 cases), and NCE biopsies (39 cases). D: Sensitivity, specificity, and PPV of normalized fluorescence intensity $>0.1$ for tumor infiltration by pathology were calculated for each time of biopsy based on all biopsies taken at and prior to that time, for the overall time-stamped sample set (73 cases) and NCE biopsies only ( 29 cases). Time was binned into 10-minute intervals post-fluorescein administration (x-axes), and the sensitivity, specificity, and PPV were averaged for all biopsies occurring up to each interval (inclusive). Gray lines indicate the time by which $50 \%$ of biopsies in each set were taken. E: The distribution times at which biopsies were acquired (minutes post-fluorescein administration) is displayed for each pathology classification: NORM 3 cases, INFILT 23 cases, TUMOR 41 cases, NEC 6 cases.

calization, or tissue pathology $(\mathrm{p}=0.611,0.357$, and 0.305 , respectively). However, an n-ANOVA interaction model of the same sample set revealed that the time between fluorescein administration and biopsy significantly influenced fluorescence intensity, but only in association with pathological diagnosis ( $\mathrm{p}=0.029$; Table 6). Indeed, while fluorescence and time post-fluorescein administration were not correlated overall (Spearman rho $=-0.07, \mathrm{p}=0.57$, 73 cases), they were anti-correlated among biopsies diagnosed as frank tumor (Spearman rho $=-0.40, \mathrm{p}=0.009$, 41 cases), and a positive but not significant correlation was seen in glioma-infiltrated tissue (Spearman rho $=0.36, \mathrm{p}=$ 0.094, 23 cases).

While a distinction in the time dependence of fluorescein signals for frank tumor and glioma-infiltrated biopsies may exist, for reasons operational (for example, highly fluorescent regions containing largely frank tumor may be the most readily targeted and thus the earliest resected), physiological (for example, pharmacokinetic distribution given different vascularization or inflammation), or biochemical (for example, oxidation, metabolic conjugation, or photobleaching in differing interstitial environments), both are considered tumor positive and are targeted for

TABLE 6. $n$-ANOVA of fractional fluorescence intensity of 73 biopsies versus time, histopathological classification, and contrast enhancement by radiographic localization

\begin{tabular}{cccc}
\hline & & \multicolumn{2}{c}{ Interaction } \\
\cline { 3 - 4 } Factor & $\begin{array}{c}\text { Linear, } \\
\mathrm{p}>\mathrm{F}\end{array}$ & $\begin{array}{c}\text { Pathological } \\
\text { Classification }\end{array}$ & $\begin{array}{c}\text { MRI } \\
\text { Localization }\end{array}$ \\
\hline $\begin{array}{c}\text { Time (mins post-fluorescein } \\
\text { administration) }\end{array}$ & 0.611 & 0.029 & 0.730 \\
\hline $\begin{array}{l}\text { Pathological classification } \\
\text { MRI localization (CE, NCE, } \\
\text { NEC, UD) }\end{array}$ & 0.305 & & 0.652 \\
\hline
\end{tabular}


resection when safe and possible. Thus, with a focus on surgical utility, we studied how the predictive utility of fluorescence intensity performed over the duration of surgery, inclusive of these variations between tumor-positive types. To determine how the time of procurement affected the clinical utility of fluorescence intensity as an indicator, for each time point at which a biopsy was procured, we calculated the sensitivity, specificity, and PPV of the 0.1 normalized fluorescence intensity threshold among samples up to and including that time point. Among the sets of both all time-stamped biopsies (73 cases; Fig. 4D upper) and NCE biopsies (29 cases; Fig. 4D lower), PPV was $>0.9$ at all time points. Most variance in these values occurred as a function of the number of samples included in the analysis; they were unstable earlier than the median time of acquisition, that is, before half of the biopsies were included, when the composition of the sample set poorly represented each pathological classification (Fig. 4E). Sensitivity of the normalized intensity threshold of 0.1 for tumor remained stable at approximately 0.7 , with a slight drop $(<10 \%)$ occurring after 170-180 minutes among all samples (Fig. 4D upper), and biopsies only in the NCE region presented a similar pattern. The robustness of the normalized fluorescence intensity threshold to time and its strong indication of tumor tissue pathology, especially in the NCE region, supports the utility of intraoperative fluorescein for guiding resection beyond the resolution of contrast enhancement and corroborates the favorable experience of surgeons in navigating the tumor margins.

\section{Discussion}

Given the prognostic importance of EOR in GBM, ${ }^{7}$ optimizing precision neuronavigational adjuncts to guide resection has become a key challenge in ensuring safe maximal reduction of the tumor burden while preserving neurological function. ${ }^{13}$ We combined intraoperative fluorescein staining with radiographically localized tissue and found the fluorescein to be a reliable marker for histopathologically abnormal tissue in both CE and NCE regions of GBM. Although adverse reactions have been reported $d^{4,5,10}$ and must be considered, our streamlined, relatively lowdose fluorescein protocol led to no fluorescein-associated adverse events or allergic reactions among our cohort of 32 patients. Importantly, implementation of fluorescein staining enhances and informs surgical judgment with the potential to facilitate safe but aggressive tumor resection that extends into the NCE tumor margin.

Gadolinium-enhanced MRI, which identifies areas of BBB disruption due to tumor pathology, is fundamental to current intraoperative stereotactic guidance in GBM. Although parenchymal fluorescein staining via intravenous administration indicates BBB disruption, ${ }^{9}$ the fluorescein intensity provides information additional to and distinct from gadolinium enhancement-in the present study, intense fluorescein staining was seen in both CE and NCE tissue, as localized by preoperative MRI. The differential permeability profiles of fluorescein and gadolinium, because of both molecular size and tumor-related pathophysiology, ${ }^{9}$ have been studied in humans and animal models but have not been quantitatively assessed beyond the $\mathrm{CE}$ boundary. Furthermore, previous comparisons of such fluorescein signal and glioma pathology $y^{1,2,9}$ have classified "gliosis or tumor cell infiltration" as "negative" and only "frank tumor"1 as "positive."

To study fluorescein signal orthogonally to radiological information, we used image analysis to quantify the fluorescein intensity of biopsy specimens and their surgical fields under Y560 illumination. Our quantitative image analysis enabled us to calculate normalized fluorescence intensity, a continuous variable, with a threshold (0.1), which showed high specificity and PPV for tumor pathology in NCE tissue (1.0). We also used this value to assess the impact of elapsed time post-fluorescein administration, presumed to be a confounder of the fluorescent signal, ${ }^{30,31}$ and observed a significant influence of time on the normalized fluorescence of only those biopsy specimens classified as frank tumor and strikingly little time dependence of the sensitivity, specificity, and PPV of the normalized fluorescence intensity threshold for tumor positivity (Fig. 4). Given the limitations of our cohort, we observed striking robustness to myriad potential time-dependent operative and biological variables-including pharmacokinetics, photobleaching, and tissue heterogeneity-particularly in nonenhancing tumor-infiltrated tissue, suggesting that the surgical utility of intraoperative fluorescein guidance extends beyond that previously documented.

It is important to note that the sensitivity and specificity measures of this study, and other studies like it, are unavoidably biased by the conditionality of surgical sampling-healthy brain tissue is not routinely resected or biopsied. Thus, fluorescein-negative, histopathologically normal tissue samples were included in this study only as a result of cases in which extensive resection, such as nondominant frontal or temporal lobectomy, was the surgical goal-and even then, it was constrained by radiological findings and other clinical and anatomical considerations. However, our results demonstrate that when combined with neuronavigation and standard neurosurgical procedures, fluorescein is a safe and reliable marker of glioma pathology. Furthermore, our observation of a fluorescence intensity threshold with strong specificity (0.1) - above which all biopsies from both CE and NCE regions showed histopathological alterations-suggests that further development of quantitative classification and its integration into real-time surgical imaging systems may facilitate resolution of both operative and biological influences on the fluorescent signal while bolstering clinical judgment beyond the boundaries of radiographic guidance.

The fluorescein signal must be interpreted in the full context of anatomical and radiological features for each patient. The additional risk of incurring neurological deficits when extending tumor resection beyond the gadolinium-defined margins requires expert surgical consideration of eloquent anatomical boundaries to achieve a safe maximal resection. The dual illumination and filter toggle of the Pentero 900/Y560 (Zeiss) instrumentation provided continuous fluorescein excitation simultaneously with a sufficiently illuminated background surgical field, allowing identification of important vascular structures and neuroanatomical landmarks, facilitating safe fluoresceinguided microsurgery based on subjective primary surgeon 
assessment. All 4 neurosurgeons found fluorescein sodium useful in the resection of all $32 \mathrm{GBM}$ resections in this cohort, citing its utility as an adjunct to neuronavigational guidance. Specifically, when normal operative events would diminish the reliability of intraoperative neuronavigation (for example, brain shift, loss of registration accuracy), fluorescein was especially beneficial in facilitating microsurgical resection of glioma tissue. In such cases, fluorescence was an additional confirmatory tool used in the decision-making process when consistent with the surgeon's clinical judgment.

The importance of safe but aggressive resection beyond the $\mathrm{CE}$ region is evidenced by both the independent predictive power of EOR on survival in $\mathrm{GBM}^{32,33}$ and the incremental improvement of overall survival (OS) with the percent of tumor volume resected, as demonstrated by Sanai et al. ${ }^{25}$ Residual tumor infiltration within $2 \mathrm{~cm}$ of the CE margin is considered the major contributor to GBM recurrence, bringing renewed attention to the potential to improve PFS and OS by extending surgical goals to address this additional tumor burden with aggressive "supratotal" resection ${ }^{11}$ beyond contrast enhancement. The demonstrated utility of fluorescein guidance in NCE regions suggests its contribution to safe microsurgical resection beyond the gadolinium-defined border. However, standard volumetric comparison solely with contrast enhancement limits true quantification of this contribution. For example, a difference in volumetric EOR (Fig. 2) was not statistically resolvable between GBM resections performed with and without fluorescein guidance in our cohort, but the removal of additional nonenhancing tumor tissue facilitated by fluorescein staining was simply not included in this traditional metric. Full evaluation of the impact of fluorescein on the EOR into the NCE regions will require both progressive, prospective radiological approaches to quantifying tumor volume and longitudinal assessment of its association with clinical outcome, which is beyond the scope of this study. Our results provide strong evidence that intraoperative fluorescein, when used judiciously in combination with surgical and neuroanatomical expertise, is a safe and reliable indicator of glioma-associated pathology, useful for guiding safe but aggressive resection in noneloquent regions, and thus may facilitate improvements in OS and PFS.

\section{Conclusions}

Glioblastoma carries a dismal prognosis despite advances in therapeutics, and safe maximal resection continues to be a mainstay of treatment and determinant of prognosis. Fluorescein sodium administration in conjunction with a dual-illumination fluorescence-filtered microscope, MRI-based neuronavigation, and traditional neurosurgical expertise enabled identification of the glioma tissue burden beyond the CE borders and facilitated safe microsurgical fluorescein-guided tumor resection. Overall, the intraoperative use of fluorescein is safe and beneficial in the resection of GBM. However, additional studies are required to elucidate the full utility of fluorescein sodium in GBM surgery. Specifically, the impact of fluorescein sodium on the extent of tumor resection is best pursued using metrics that reflect on nonenhancing tumor infiltra- tion, and its impact on patient survival must be evaluated further in longitudinal cohorts. The application of fluorescein sodium to glioma resection is inexpensive and safe and may help surgeons achieve increased maximal safe resection in patients.

\section{Acknowledgments}

We express our appreciation to the operating room nursing staff at Columbia University Medical Center.

\section{References}

1. Acerbi F, Broggi M, Eoli M, Anghileri E, Cavallo C, Boffano $\mathrm{C}$, et al: Is fluorescein-guided technique able to help in resection of high-grade gliomas? Neurosurg Focus 36(2):E5, 2014

2. Acerbi F, Broggi M, Eoli M, Anghileri E, Cuppini L, Pollo B, et al: Fluorescein-guided surgery for grade IV gliomas with a dedicated filter on the surgical microscope: preliminary results in 12 cases. Acta Neurochir (Wien) 155:1277-1286, 2013

3. Albert FK, Forsting M, Sartor K, Adams HP, Kunze S: Early postoperative magnetic resonance imaging after resection of malignant glioma: objective evaluation of residual tumor and its influence on regrowth and prognosis. Neurosurgery 34:45-61, 1994

4. Balbino M, Silva G, Correia GCTP: Anaphylaxis with convulsions following intravenous fluorescein angiography at an outpatient clinic. Einstein (São Paulo) 10:374-376, 2012

5. Bearelly S, Rao S, Fekrat S: Anaphylaxis following intravenous fluorescein angiography in a vitreoretinal clinic: report of 4 cases. Can J Ophthalmol 44:444-445, 2009

6. Berger MS: Malignant astrocytomas: surgical aspects. Semin Oncol 21:172-185, 1994

7. Bloch O, Han SJ, Cha S, Sun MZ, Aghi MK, McDermott MW, et al: Impact of extent of resection for recurrent glioblastoma on overall survival: clinical article. J Neurosurg 117:1032-1038, 2012

8. Chow DS, Qi J, Guo X, Miloushev VZ, Iwamoto FM, Bruce JN, et al: Semiautomated volumetric measurement on postcontrast MR imaging for analysis of recurrent and residual disease in glioblastoma multiforme. AJNR Am J Neuroradiol 35:498-503, 2014

9. Diaz RJ, Dios RR, Hattab EM, Burrell K, Rakopoulos P, Sabha N, et al: Study of the biodistribution of fluorescein in glioma-infiltrated mouse brain and histopathological correlation of intraoperative findings in high-grade gliomas resected under fluorescein fluorescence guidance. J Neurosurg 122:1360-1369, 2015

10. Dilek O, Ihsan A, Tulay H: Anaphylactic reaction after fluorescein sodium administration during intracranial surgery. $\mathbf{J}$ Clin Neurosci 18:430-431, 2011

11. Duffau H: Is supratotal resection of glioblastoma in noneloquent areas possible? World Neurosurg 82:e101-e103, 2014

12. Eléouet S, Rousset N, Carré J, Bourré L, Vonarx V, Lajat Y, et al: In vitro fluorescence, toxicity and phototoxicity induced by delta-aminolevulinic acid (ALA) or ALA-esters. Photochem Photobiol 71:447-454, 2000

13. Fecci PE, Babu R, Adamson DC, Sampson JH: Editorial: Turning fluorescence into black and white. J Neurosurg 122:1356-1358, 2015

14. Hardesty DA, Sanai N: The value of glioma extent of resection in the modern neurosurgical era. Front Neurol 3:140, 2012

15. Kamp MA, Felsberg J, Sadat H, Kuzibaev J, Steiger HJ, Rapp $\mathrm{M}$, et al: 5-ALA-induced fluorescence behavior of reactive tissue changes following glioblastoma treatment with radia- 
tion and chemotherapy. Acta Neurochir (Wien) 157:207214,2015

16. Koc K, Anik I, Cabuk B, Ceylan S: Fluorescein sodiumguided surgery in glioblastoma multiforme: a prospective evaluation. Br J Neurosurg 22:99-103, 2008

17. Kuroiwa T, Kajimoto Y, Ohta T: Development of a fluorescein operative microscope for use during malignant glioma surgery: a technical note and preliminary report. Surg Neurol 50:41-49, 1998

18. Marko NF, Weil RJ, Schroeder JL, Lang FF, Suki D, Sawaya RE: Extent of resection of glioblastoma revisited: personalized survival modeling facilitates more accurate survival prediction and supports a maximum-safe-resection approach to surgery. J Clin Oncol 32:774-782, 2014

19. McGirt MJ, Chaichana KL, Gathinji M, Attenello FJ, Than $\mathrm{K}$, Olivi A, et al: Independent association of extent of resection with survival in patients with malignant brain astrocytoma. J Neurosurg 110:156-162, 2009

20. Moore GE: Fluorescein as an agent in the differentiation of normal and malignant tissues. Science 106:130-131, 1947

21. Moore GE, Peyton WT, French LA, Walker WW: The clinical use of fluorescein in neurosurgery; the localization of brain tumors. J Neurosurg 5:392-398, 1948

22. Okuda T, Kataoka K, Yabuuchi T, Yugami H, Kato A: Fluorescence-guided surgery of metastatic brain tumors using fluorescein sodium. J Clin Neurosci 17:118-121, 2010

23. Rey-Dios R, Cohen-Gadol AA: Technical principles and neurosurgical applications of fluorescein fluorescence using a microscope-integrated fluorescence module. Acta Neurochir (Wien) 155:701-706, 2013

24. Rey-Dios R, Hattab EM, Cohen-Gadol AA: Use of intraoperative fluorescein sodium fluorescence to improve the accuracy of tissue diagnosis during stereotactic needle biopsy of high-grade gliomas. Acta Neurochir (Wien) 156:1071-1075, 2014

25. Sanai N, Polley MY, McDermott MW, Parsa AT, Berger MS: An extent of resection threshold for newly diagnosed glioblastomas. J Neurosurg 115:3-8, 2011

26. Schebesch KM, Proescholdt M, Höhne J, Hohenberger C, Hansen E, Riemenschneider MJ, et al: Sodium fluoresceinguided resection under the YELLOW $560 \mathrm{~nm}$ surgical microscope filter in malignant brain tumor surgery-a feasibility study. Acta Neurochir (Wien) 155:693-699, 2013

27. Shinoda J, Yano H, Yoshimura S, Okumura A, Kaku Y, Iwama T, et al: Fluorescence-guided resection of glioblastoma multiforme by using high-dose fluorescein sodium. Technical note. J Neurosurg 99:597-603, 2003

28. Shoolingin-Jordan PM, Al-Daihan S, Alexeev D, Baxter RL, Bottomley SS, Kahari ID, et al: 5-Aminolevulinic acid synthase: mechanism, mutations and medicine. Biochim Biophys Acta 1647:361-366, 2003

29. Sjöback R, Nygren J, Kubista M: Absorption and fluorescence properties of fluorescein. Spectrochim Acta A Mol Biomol Spectrosc 51:L7-L21, 1995
30. Stummer W: Factors confounding fluorescein-guided malignant glioma resections: edema bulk flow, dose, timing, and now: imaging hardware? Acta Neurochir (Wien) 158:327328,2016

31. Stummer W: Fluorescein in brain metastasis and glioma surgery. Acta Neurochir (Wien) 157:2199-2200, 2015

32. Stummer W, Pichlmeier U, Meinel T, Wiestler OD, Zanella F, Reulen HJ: Fluorescence-guided surgery with 5-aminolevulinic acid for resection of malignant glioma: a randomised controlled multicentre phase III trial. Lancet Oncol 7:392 401, 2006

33. Stummer W, Reulen HJ, Meinel T, Pichlmeier U, Schumacher W, Tonn JC, et al: Extent of resection and survival in glioblastoma multiforme: identification of and adjustment for bias. Neurosurgery 62:564-576, 2008

34. Stupp R, Mason WP, van den Bent MJ, Weller M, Fisher $\mathrm{B}$, Taphoorn MJ, et al: Radiotherapy plus concomitant and adjuvant temozolomide for glioblastoma. N Engl J Med 352:987-996, 2005

35. Wallner KE, Galicich JH, Krol G, Arbit E, Malkin MG: Patterns of failure following treatment for glioblastoma multiforme and anaplastic astrocytoma. Int J Radiat Oncol Biol Phys 16:1405-1409, 1989

\section{Disclosures}

Dr. Neira has received travel monies from Carl Zeiss. Dr. Canoll is supported by NIH/NINDS Grant No. R01NS066955. A Zeiss Pentero 900 microscope with Yellow 560 Filter was received from Carl Zeiss Meditec AG.

\section{Author Contributions}

Conception and design: Bruce, Neira, Ung, Canoll. Acquisition of data: Bruce, Neira, Ung, Malone, Chow, Samanamud, Zanazzi, Guo, Zhao, Sheth, McKhann, Sisti, Canoll, D'Amico. Analysis and interpretation of data: Bruce, Neira, Ung, Sims, Malone, Chow, Zanazzi, Guo, Zhao, Canoll, D’Amico. Drafting the article: Bruce, Neira, Ung, Sims. Critically revising the article: Bruce, Neira, Ung, Sims, Malone, Samanamud, Canoll, D'Amico. Reviewed submitted version of manuscript: all authors. Approved the final version of the manuscript on behalf of all authors: Bruce. Statistical analysis: Neira, Ung, Sims, Chow, Guo, Zhao. Administrative/technical/material support: Bruce, Samanamud, Zanazzi, Guo, Bowden, Sheth, McKhann, Sisti, Canoll. Study supervision: Bruce, Sims, Malone, Sheth, McKhann, Sisti, Canoll, D’Amico.

\section{Correspondence}

Jeffrey N. Bruce, Department of Neurological Surgery, Columbia University Medical Center, 710 W 168th St., Rm. 434, New York, NY 10032. email: jnb2@columbia.edu. 